\title{
Monitoring of Thermal Damage after Deposition of Coatings via Barkhausen Noise Technique
}

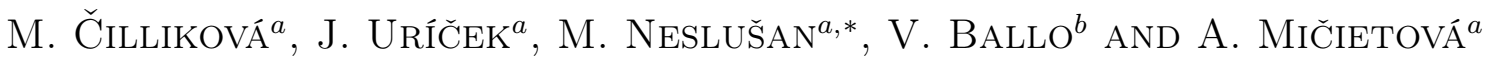 \\ ${ }^{a}$ University of Žilina, Univerzitná 1, 01026 Žilina, Slovakia \\ ${ }^{b}$ Staton, s.r.o., Sadová 1148, 03853 Turany, Slovakia
}

\begin{abstract}
This paper deals with monitoring of thermal damage of hardened steel 100Cr during deposition of coatings via magnetic Barkhausen noise. This study investigates influence of the different deposition regimes when the samples of the different hardness are coated. Hardness of the matrix before and after deposition is correlated with the Barkhausen noise features extracted from the raw Barkhausen noise signal. Results indicate that the Barkhausen noise technique is suitable for monitoring surface over-tempering initiated during coating deposition. Moreover, position of the Barkhausen noise envelope maximum correlates with hardness of the sample more than conventional effective value.
\end{abstract}

DOI: 10.12693/APhysPolA.137.637

PACS/topics: coatings, Barkhausen noise, thermal softening

\section{Introduction}

Magnetic Barkhausen noise (MBN) technique is widely employed for monitoring ground surfaces in the real industrial applications. The low MBN values for untouched surfaces are in contrast with the high MBN emission due to residual stress alterations and thermal overtempering during grinding [1]. MBN is a product of irreversible discontinuous Bloch walls (BWs) motion during cyclic magnetization. BWs interfere with stress state, as well as microstructure features (such as dislocations, carbides, grain boundaries, non-ferromagnetic particles, etc.) which pin BWs motion [2]. The high MBN magnitude of over-tempered surfaces after grinding is mainly associated with reduced dislocations and carbides density thermally initiated by elevated temperatures which correspond with the decreased pinning strength [2].

Nowadays, some critical parts after hardening undergo the consecutive coating process in which thin nearsurface layer enhances the functionality of component. Deposition process can be performed at the different regimes (especially temperatures) on the components of variable hardness. On one hand, coating can improve some component properties such as corrosion resistance, friction coefficient, etc. $[3,4]$. On the other hand, deposition of coating is the process carried out at elevated temperatures and the component matrix could be overtempered. Furthermore, remarkable non- homogeneity of coating thickness or/and underlying matrix tempering can be found especially for components of complicated geometry. For this reason, a nondestructive technique capable to reveal unacceptable surface state would be beneficial. As it was above mentioned, MBN technique

\footnotetext{
* corresponding author; e-mail:

miroslav.neslusan@fstroj.uniza.sk
}

is sensitive to the over-tempering of hardened components and this pilot study investigates potential of MBN for monitoring components of variable hardness coated at the different deposition regimes.

\section{Experiments}

The experimental study was carried out on the bearing steel $100 \mathrm{Cr} 6$ heat treated on variable hardness $41,54,59$, and $61.5 \mathrm{HRC}$. The samples of dimension $17 \times 17 \times 5 \mathrm{~mm}^{3}$ were quenched from temperature $830^{\circ} \mathrm{C}$ in the oil of temperature of $62^{\circ} \mathrm{C}$ and tempered for $2 \mathrm{~h}$ afterwards (tempering temperature: $160^{\circ} \mathrm{C}$ for hardness $61.5 \mathrm{HRC}$, $310^{\circ} \mathrm{C}$ for hardness $54 \mathrm{HRC}, 380^{\circ} \mathrm{C}$ for hardness $59 \mathrm{HRC}$, and $530^{\circ} \mathrm{C}$ for hardness $41 \mathrm{HRC}$ ). Each series of hardness consists of 6 samples. 3 samples undergo the low temperature deposition and the 3 samples - the high temperature deposition regimes. The low temperature regime (LTR) parameters were magnetosputtering method, temperature of deposition $180^{\circ} \mathrm{C}$, WC coating of thickness approximately $1 \mu \mathrm{m}$ (see Fig. 1a), hardness $17.5 \pm 1 \mathrm{GPa}$, friction coefficient 0.1 , deposition pressure $0.5 \mathrm{~Pa}$. The high temperature regime (HTR) parameters were arc evaporation method, temperature

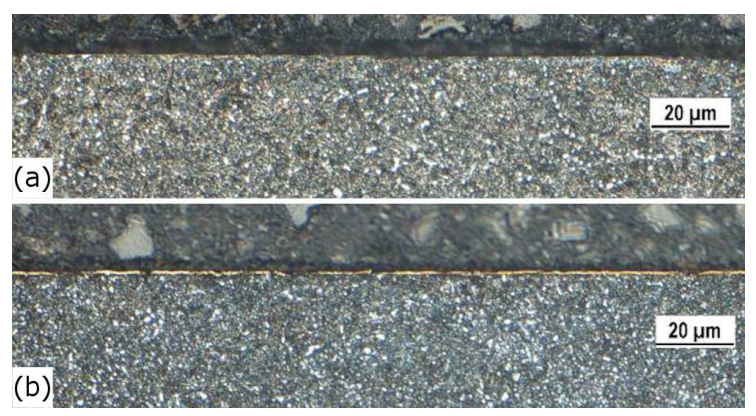

Fig. 1. (a) Micrograph of surface, low temperature deposition. (b) Micrograph of surface, high temperature deposition. 
of deposition $450^{\circ} \mathrm{C}$, TiN coating of thickness approximately $2 \mu \mathrm{m}$ (see Fig. 1b), hardness $21.5 \pm 1.5 \mathrm{GPa}$, friction coefficient 0.6 , deposition pressure $2 \mathrm{~Pa}$.

To reveal the microstructure of the surface the $15 \mathrm{~mm}$ long pieces were prepared for metallographic observations (etched by 3\% Nital for $10 \mathrm{~s}$ ). The Rockwell hardness was measured by the use of $\mathrm{RB}-1 / \mathrm{PC}$ with load 95-101 N for $10 \mathrm{~s}$. MBN was measured by the use of $\mu$ Scan 500 (magnetizing voltage $16 \mathrm{~V}$, magnetizing frequency $125 \mathrm{~Hz}, 10$ bursts, frequency range of MBN from 10 to $1000 \mathrm{kHz}$ ). MBN refers to the root-mean-square (rms, effective) value of the signal.

\section{Results of experiments}

Figure 2 shows that the matrix hardness stays nearly untouched after LTR whereas HTR initiates remarkable hardness decrease for samples of higher hardness. This figure also demonstrates that hardness decrease occurs as soon as the temperature of deposition in the chamber considerably exceeds tempering temperatures in the furnace during heat treatment. Difference of hardness before and after deposition becomes more remarkable with the increasing difference between tempering and deposition temperatures. The lift-off due to the coating layer tends to drop down MBN. However, the thickness of coatings is very low and properties of the matrix takes the major role.

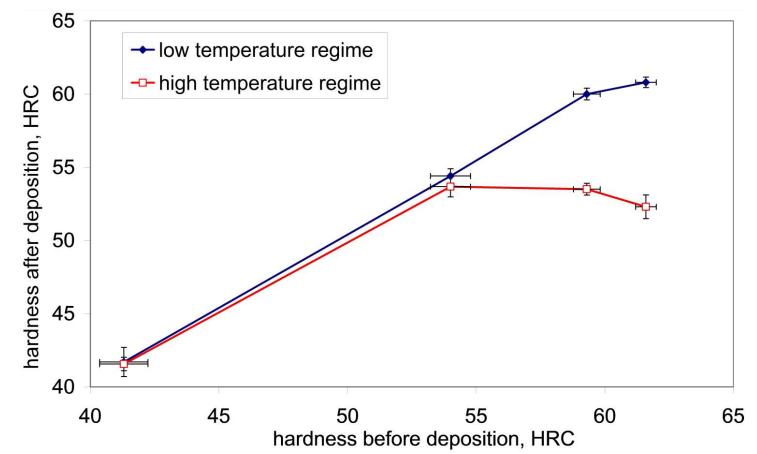

Fig. 2. Influence of coating deposition regime on sample hardness.

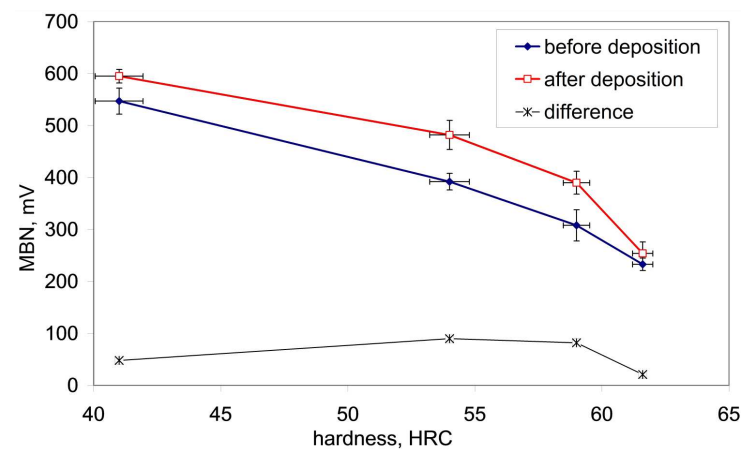

Fig. 3. MBN before and after LTR.

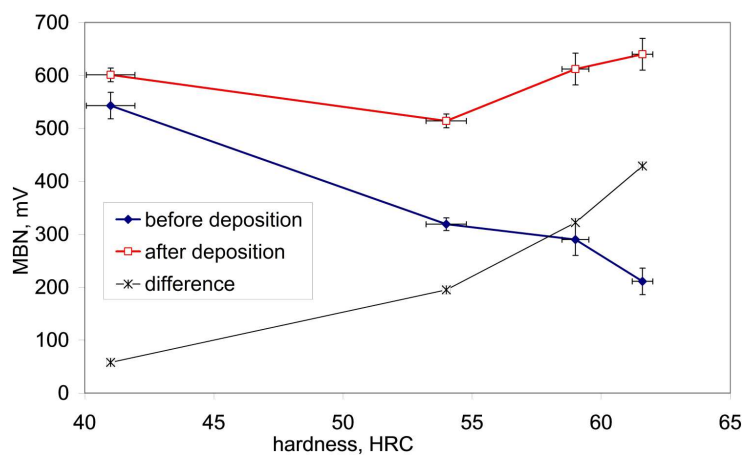

Fig. 4. MBN before and after HTR.

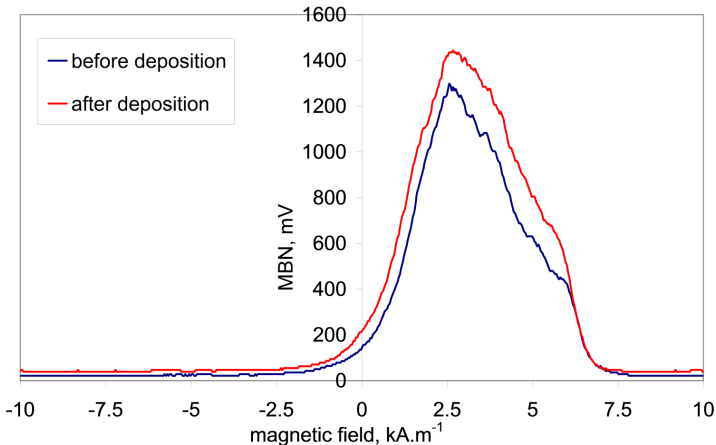

Fig. 5. MBN envelopes, LTR, 41 HRC.

Hardness of bearing steel is mainly a function of dislocation density, as well as carbides density and their distribution [2]. BWs interfere with dislocations and carbides and higher MBN values for samples of lower hardness are due to reduced dislocation density whereas carbides stay nearly untouched. Therefore, the high MBN corresponds to the low mechanical hardness of the sample whereas the high MBN should be associated with the considerable thermal over-tempering. Moreover, decreasing volume of retained austenite also contributes to the higher MBN for softer samples after heat treatment. Figures 3 and 4 demonstrate that $\mathrm{MBN}$ values correlate with the matrix hardness before and after deposition. LTR initiates only moderate decrease of MBN whereas the differences between MBN before and after HTR increase along with sample hardness. Figures $2-4$ clearly indicate that HTR initiates unacceptable thermal over-tempering of the matrix whereas high hardness can be preserved applying LTR.

MBN envelopes (as those shown in Figs. 5 and 6) also clearly demonstrate that peak position retains nearly untouched after LTR whereas MBN envelope after HTR is remarkably shifted to the weaker magnetic fields. Cumulative evolution of peak position before and after deposition is illustrated in Fig. 7. This figure also demonstrates that peak position is sensitive enough against the thermal tempering. Peak position strongly correlates with magnetic hardness of a body. 


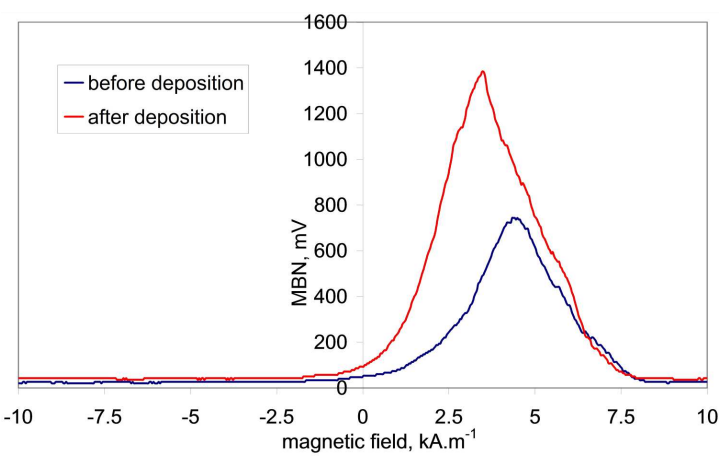

Fig. 6. MBN envelopes, HTR, 61.5 HRC.

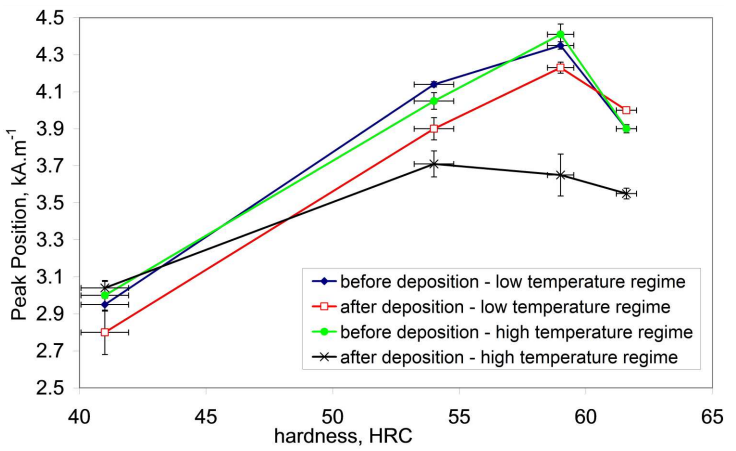

Fig. 7. Evolution of peak position.

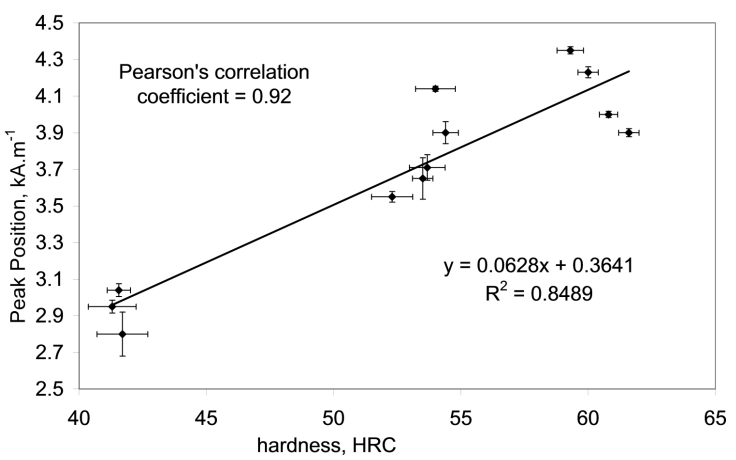

Fig. 8. Sample hardness versus peak position.

On the one hand, peak position increases along with increasing hardness of the samples before coating deposition and after tempering during heat treatment in the furnace. However, this evolution exhibits certain drop for the samples of hardness $61.5 \mathrm{HRC}$ (see Fig. 7). On the other hand, peak position correlates much better with the decreasing hardness of the samples especially after HTR. Figures 8 and 9 and the corresponding calculations show that peak position MBN feature is more sensitive to the thermal over-tempering initiated by deposition of coating under the different regimes than the conventional MBN parameter. Industrial application of MBN technique

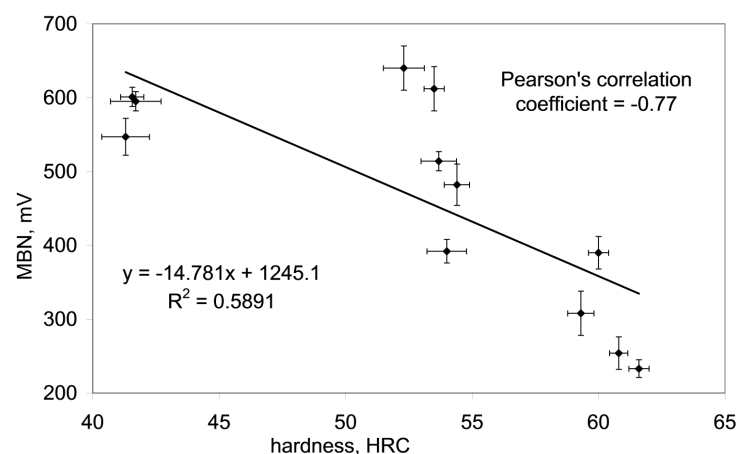

Fig. 9. Sample hardness versus MBN.

requires finding reliable correlation between conventional information about component state and the corresponding MBN signal (or extracted features). Components state analyzed in a conventional destructive manner in this particular case is expressed by the matrix hardness. Peak position very often expresses magnetic hardness of a body which correlates with its mechanical hardness [5]. Such aspect explains why peak position is more sensitive to the decreased matrix hardness after deposition of coating than the conventional MBN effective value.

\section{Conclusions}

The possible concept in which thermal damage initiated by deposition of coatings could be monitored is driven by relation between MBN (extracted features) and hardness of the matrix underlying beneath the coating. Sensitivity of the sample against the over-tempering during deposition is a function mainly of sample hardness, as well as the regime of coating deposition.

\section{Acknowledgments}

The authors acknowledge the financial support provided by the VEGA project $1 / 0121 / 17$ and KEGA project no. $008 Z ̌ U-4 / 2018$.

\section{References}

[1] V. Moorthy, B.A. Shaw, P. Mountford, P. Hopkins, Acta Mater. 53, 4997 (2005).

[2] M. Neslušan, J. Čížek, K. Kolařík, P. Minárik, M. Cilliková, O. Melikhová, J. Mater. Process. Technol. 240, 104 (2017).

[3] F. Wang, F. Zhang, L. Zheng, H. Zhang, Appl. Surf. Sci. 423, 695 (2017).

[4] N. Ley, S.S. Joshi, B. Zhang, Y. Ho, N.B. Dahotre, M.L. Young, Surf. Coat. Technol. 348, 150 (2018).

[5] A. Sorsa, K. Leviskä, S. Santa-aho, T. Lepistö, NDTE Int. 46, 100 (2012). 